\title{
A NOTE ON THE NUMBER OF PRIMES IN SHORT INTERVALS
}

\author{
D. A. GOLDSTON AND S. M. GONEK \\ (Communicated by William Adams)
}

\begin{abstract}
Let $J(\beta, T)=\int_{1}^{T^{\beta}}\left(\sum_{x<p^{k} \leq x+x / T} \log p-x / T\right)^{2} d x / x^{2}$, where the sum is over prime powers. H. L. Montgomery has shown that on the Riemann hypothesis, there is a positive constant $C_{0}$ such that for each $\beta \geq 1, J(\beta, T) \leq$ $C_{0} \beta \log ^{2} T / T$, provided that $T$ is sufficiently large. Here we prove a slightly stronger result from which we deduce a lower bound of the same order.
\end{abstract}

\section{INTRODUCTION}

In 1943 A. Selberg [7] proved that if the Riemann hypothesis $(\mathrm{RH})$ is true, then

$$
\begin{aligned}
J(\beta, T) & =\int_{1}^{T^{\beta}}\left(\psi\left(x+\frac{x}{T}\right)-\psi(x)-\frac{x}{T}\right)^{2} x^{-2} d x \\
& \ll_{\beta} \frac{\log ^{2} T}{T}
\end{aligned}
$$

for fixed $\beta \geq 1$ and $T \geq 2$; here $\psi(x)=\sum_{n \leq x} \Lambda(n)$, where $\Lambda(n)=\log p$ if $n=p^{m}$ with $p$ a prime number and $m \geq 1$, and $\Lambda(n)=0$ otherwise. H. L. Montgomery (unpublished) later made the $\beta$ dependence explicit by proving that on RH there exists an absolute constant $C_{0}$ such that, for each $\beta \geq 1$,

$$
J(\beta, T) \leq C_{0} \frac{\beta \log ^{2} T}{T}
$$

as $T \rightarrow \infty$. Proofs of this subsequently appeared in [1], [5], and [4]. Our object here is to prove a stronger result for $J(\beta, T)$ on $\mathrm{RH}$ which immediately implies (1) and, moreover, shows that apart from constants (1) is best possible.

We shall use the standard symbols $\ll, \gg O, O$, and $\sim$ and, unless otherwise indicated, all implied constants will be absolute.

Theorem. Assume the Riemann hypothesis. Then there are absolute constants $C_{2}>C_{1}>0$ such that for each $\beta>0$,

$$
C_{1} \frac{\log ^{2} T}{T} \leq J(\beta+2, T)-J(\beta, T) \leq C_{2} \frac{\log ^{2} T}{T}
$$

for all sufficiently large $T$.

Received by the editors March 13, 1989.

1980 Mathematics Subject Classification (1985 Revision). Primary 11M26, 11 N05.

Research of both authors was partially supported by grants from the National Science Foundation.

(C) 1990 American Mathematical Society $0002-9939 / 90 \$ 1.00+\$ .25$ per page 
Corollary. Assume the Riemann hypothesis. Then there are absolute constants $D_{2}>D_{1}>0$ such that, for each $\beta \geq 1$,

$$
D_{1} \frac{\beta \log ^{2} T}{T} \leq J(\beta, T) \leq D_{2} \frac{\beta \log ^{2} T}{T}
$$

for all sufficiently large $T$.

The Theorem should be compared with a result of Gallagher and Mueller [1] (also see [3]) which asserts that RH and the pair correlation conjecture together imply that for fixed $\beta_{1}>\beta_{0} \geq 1$,

$$
J\left(\beta_{1}, T\right)-J\left(\beta_{0}, T\right)=\left(\left(\beta_{1}-\beta_{0}\right)+o(1)\right) \frac{\log ^{2} T}{T} \quad(\text { as } T \rightarrow \infty) .
$$

Since for $0<\beta \leq 1$ one also has (unconditionally) that

$$
J(\beta, T) \sim \frac{\beta^{2}}{2} \frac{\log ^{2} T}{T} \quad(\text { as } T \rightarrow \infty)
$$

(see [1]), we see that on the above hypotheses

$$
J(\beta+2, T)-J(\beta, T) \sim \begin{cases}\left(3 / 2+\beta-\beta^{2} / 2\right) \frac{\log ^{2} T}{T} & \text { if } 0<\beta \leq 1, \\ 2 \frac{\log ^{2} T}{T} & \text { if } \beta \geq 1 .\end{cases}
$$

Our proof will actually show that if $\beta>0$, then

$$
.3 \frac{\log ^{2} T}{T} \leq J(\beta+2, T)-J(\beta, T) \leq 21.65 \frac{\log ^{2} T}{T}
$$

for all sufficiently large $T$. It is also possible by our method to show that

$$
\left(\beta_{1}-\beta_{0}\right) \frac{\log ^{2} T}{T} \ll J\left(\beta_{1}, T\right)-J\left(\beta_{0}, T\right) \ll\left(\beta_{1}-\beta_{0}\right) \frac{\log ^{2} T}{T}
$$

for $\beta_{1}>\beta_{0}>0$ as long as $\beta_{1}-\beta_{0}>6-2 \sqrt{6}=1.10102 \ldots$ It is doubtful, however, whether one can obtain this for arbitrarily small differences $\beta_{1}-\beta_{0}$ on RH alone.

\section{A LEMMA}

We prove the Theorem by relating $J(\beta, T)$ to averages of the function

$$
F(\alpha, T)=\left(\frac{T}{2 \pi} \log T\right)^{-1} \sum_{0<\gamma, \gamma^{\prime} \leq T} T^{i \alpha\left(\gamma-\gamma^{\prime}\right)} w\left(\gamma-\gamma^{\prime}\right)
$$

introduced by Montgomery [6]; here $\alpha$ is real, $T \geq 2, w(u)=4 / 4+u^{2}$, and $\gamma, \gamma^{\prime}$ denote the imaginary parts of zeros of the Riemann zeta-function. We shall then require the following result which generalizes and strengthens Lemma A of [2]. 
Lemma. Assume the Riemann hypothesis and let

$$
G(\alpha, T)=\left(\frac{T}{2 \pi} \log T\right)^{-1} \sum_{0<\gamma, \gamma^{\prime} \leq T}\left(\frac{\sin \frac{\alpha}{2}\left(\gamma-\gamma^{\prime}\right) \log T}{\frac{\alpha}{2}\left(\gamma-\gamma^{\prime}\right) \log T}\right)^{2} w\left(\gamma-\gamma^{\prime}\right) .
$$

Then for $a>0, \beta$ real, and $T \geq 2$,

(3) $a\left(1-\frac{1}{2} G\left(\frac{a}{2}, T\right)\right) \leq \int_{\beta}^{\beta+a} F(\alpha, T) d \alpha \leq a\left(G(a, T)+\frac{1}{2} G\left(\frac{a}{2}, T\right)\right)$.

Proof. The proof of the lower bound in Lemma A (which corresponds to $a=2$ here) extends in a straightforward way to give the lower bound in (3).

On the other hand, the upper bound in Lemma A generalizes to $2 a G(a, T)$ which is not as good as the bound in (3).

To obtain the present upper bound define $K_{b}(u)=\max (1-|u| / b, 0), b>0$, and consider the function

$$
R_{a}(u)=K_{a}(u)+\frac{1}{2} K_{a / 2}(u-a / 2)+\frac{1}{2} K_{a / 2}(u+a / 2) .
$$

Defining the Fourier transform of $f(x)$ by

$$
\hat{f}(\omega)=\int_{-\infty}^{\infty} f(x) e(-x \omega) d x
$$

where $e(u)=e^{2 \pi i u}$, we have that

$$
\widehat{K}_{b}(\omega)=b\left(\frac{\sin \pi b \omega}{\pi b \omega}\right)^{2}
$$

Thus

$$
\widehat{R}_{a}(\omega)=a\left\{\left(\frac{\sin \pi a \omega}{\pi a \omega}\right)^{2}+\frac{1}{2} \cos \pi a \omega\left(\frac{\sin \pi a \omega / 2}{\pi a \omega / 2}\right)^{2}\right\} .
$$

Now clearly $R_{a}(u) \geq 0$ for all $u$, and $R_{a}(u)=1$ for $|u| \leq a / 2$. Furthermore (see [5]), $F(\alpha, T) \geq 0$. Hence

$$
\begin{aligned}
\int_{\beta}^{\beta+a} F(\alpha, T) d \alpha= & \int_{(\beta+a / 2)-a / 2}^{(\beta+a / 2)+a / 2} F(\alpha, T) d \alpha \\
\leq & \int_{-\infty}^{\infty} F(\alpha, T) R_{a}(\alpha-(\beta+a / 2)) d \alpha \\
= & \left(\frac{T}{2 \pi} \log T\right)^{-1} \sum_{0<\gamma, \gamma^{\prime} \leq T} T^{i(\beta+a / 2)\left(\gamma-\gamma^{\prime}\right)} \widehat{R}_{a}\left(\frac{\left(\gamma-\gamma^{\prime}\right) \log T}{2 \pi}\right) \\
& \times w\left(\gamma-\gamma^{\prime}\right) \\
\leq & a\left(G(a, T)+\frac{1}{2} G(a / 2, T)\right) .
\end{aligned}
$$

This proves the result. 
We require the lower bound of the lemma for $a=2-\eta$ and the upper bound for $a=2+\eta$, where $\eta>0$. Montgomery [6] has shown that on $\mathrm{RH}$,

$$
G(\alpha, T) \sim\left(\frac{1}{\alpha}+\frac{\alpha}{3}\right)
$$

for $0<\alpha \leq 1$ as $T \rightarrow \infty$. Hence

$$
\int_{\beta}^{\beta+2-\eta} F(\alpha, T) d \alpha \geq(1+o(1))\left(\frac{2}{3}-c_{1}(\eta)\right) \quad(\text { as } T \rightarrow \infty),
$$

where $c_{1}(\eta) \rightarrow 0$ as $\eta \rightarrow 0^{+}$. For the upper bound we use the inequality

$$
G(j+\eta, T) \leq 4 / 3+c_{2}(\eta)+o(1)
$$

as $T \rightarrow \infty(j=1$ or 2$)$, where $c_{2}(\eta) \rightarrow 0$ as $\eta \rightarrow 0^{+}$; these follow from Lemma 7 of [2]. We then obtain

$$
\int_{\beta}^{\beta+2+\eta} F(\alpha, T) d \alpha \leq(1+o(1))\left(4+c_{3}(\eta)\right) \quad(\text { as } T \rightarrow \infty),
$$

where $c_{3}(\eta) \rightarrow 0$ as $\eta \rightarrow 0^{+}$.

\section{PROOF OF THE THEOREM AND COROLLARY}

We begin by quoting two results from Goldston [3]. We remind the reader that the Riemann hypothesis is assumed throughout this section.

Let $g(x)$ be a complex valued function such that $g(x) \ll\left(1+x^{2}\right)^{-1}, \hat{g}(\omega) \ll$ $\left(1+\omega^{2}\right)^{-1}$, and $\hat{g}(\omega)=0$ for $\omega \leq 0$, and define

$$
H_{ \pm}(\mu, U)=\int_{0}^{U}\left|\sum_{\gamma} g( \pm(t-\gamma) \mu)\right|^{2} d t
$$

where the sum is over the ordinates of the nontrivial zeros of $\zeta(s)$. Then by Equations (5.2) and (5.3) of [3],

$$
H_{ \pm}(\mu, U)=U \int_{1}^{\infty} F(\alpha, U)|\hat{g}(\alpha)|^{2} d \alpha+o(U)
$$

for $|\mu-1 / 2 \pi \log U| \leq C \log \log U$, where $C$ is an arbitrary positive constant. Furthermore, by Lemma 5 of [3] ${ }^{1}$

$$
\begin{aligned}
\int_{0}^{\infty}( & \left.\left(e^{u+\delta}\right)-\psi\left(e^{l:}\right)-\left(e^{\delta}-1\right) e^{u}\right)^{2} e^{-u}\left|\hat{g}\left(\frac{u}{2 \pi \nu}\right)\right|^{2} d u \\
= & 8 \pi \nu^{2} \int_{-\infty}^{\infty}\left(\frac{\sin \frac{\delta}{2} t}{t}\right)^{2}\left|\sum_{\gamma} g((t-\gamma) \nu)\right|^{2} d t \\
& +O(\delta)+O\left(\nu^{2} \delta^{3 / 2} \log 1 / \delta\right)
\end{aligned}
$$

${ }^{1}$ Lemma 5 contains two typographical errors: " $T \geq 2$ " should be removed and the factor $\left(\psi\left(e^{u+\delta}\right)-\psi\left(e^{u}\right)-\left(e^{\delta}-1\right) e^{u}\right)$ in $(4.2)$ should be squared. 
uniformly for $\nu \geq 1$ and $0<\delta \leq 1 / 4$. If we set $e^{\delta}=1+1 / T, \nu=1 / 2 \pi \log T$, and $x=e^{u}$, we may rewrite this as

$$
\begin{aligned}
& \int_{1}^{\infty}\left(\psi\left(x+\frac{x}{T}\right)-\psi(x)-\frac{x}{T}\right)^{2}\left|\hat{g}\left(\frac{\log x}{\log T}\right)\right|^{2} \frac{d x}{x^{2}} \\
&=\frac{2}{\pi} \log ^{2} T \int_{0}^{\infty}\left(\frac{\sin \frac{\delta}{2} t}{t}\right)^{2}\left(\left|\sum_{\gamma} g\left((t-\gamma) \frac{\log T}{2 \pi}\right)\right|^{2}\right. \\
&\left.+\left|\sum_{\gamma} g\left((\gamma-t) \frac{\log T}{2 \pi}\right)\right|^{2}\right) d t+O\left(\frac{1}{T}\right) .
\end{aligned}
$$

We now choose a pair of functions $g, \hat{g}$ for which the above conditions hold and such that $\hat{g}$ approximates the characteristic function of the interval $[\beta, \beta+b]$ from below, with $\beta>0$. More specifically, we take $\hat{g}=0$ off of $[\beta, \beta+b], \hat{g}=1$ on $[\beta+\eta, \beta+b-\eta]$, and $|\hat{g}| \leq 1$ otherwise, where $0<\eta<b / 2$ is fixed. (Such a pair may be constructed explicitly by a linear change of variable from the pair defined in (3.6) of [3].) With this choice of $\hat{g}$ in (7) we immediately obtain

(9) $U \int_{\beta+\eta}^{\beta+b-\eta} F(\alpha, U) d \alpha+o(U) \leq H_{ \pm}(\mu, U) \leq U \int_{\beta}^{\beta+b} F(\alpha, U) d \alpha+o(U)$

for $|\mu-1 / 2 \pi \log U| \leq C \log \log U$. The same choice in the left-hand side of (8) leads to the inequalities

$$
\begin{aligned}
J(\beta & +b-\eta, T)-J(\beta+\eta, T) \\
& \leq \int_{1}^{\infty}\left(\psi\left(\frac{x}{T}\right)-\psi(x)-\frac{x}{T}\right)^{2}\left|\hat{g}\left(\frac{\log x}{\log T}\right)\right|^{2} \frac{d x}{x^{2}} \\
& \leq J(\beta+b, T)-J(\beta, T) .
\end{aligned}
$$

We now obtain the lower bound of the theorem. Taking $b=2$ in (10) and using $(8)$, we see that

$$
\begin{aligned}
& J(\beta+2, T)-J(\beta, T) \\
& \geq \frac{2}{\pi} \log ^{2} T \int_{0}^{\theta T}\left(\frac{\sin \frac{\delta}{2} t}{t}\right)^{2}\left\{\left|\sum_{\gamma} g\left((t-\gamma) \frac{\log T}{2 \pi}\right)\right|^{2}\right. \\
&\left.+\left|\sum_{\gamma} g\left((\gamma-t) \frac{\log T}{2 \pi}\right)\right|^{2}\right\} d t+O\left(\frac{1}{T}\right),
\end{aligned}
$$

where $0<\theta<\pi$. Now $(\sin (\delta t / 2) / t)^{2}$ is monotone decreasing for $0 \leq t \leq \theta T$, so by (6) this is

$$
\geq \frac{2}{\pi} \log ^{2} T\left(\frac{\sin \frac{\delta}{2} \theta T}{\theta T}\right)^{2}\left\{H_{+}\left(\frac{\log T}{2 \pi}, \theta T\right)+H_{-}\left(\frac{\log T}{2 \pi} \theta T\right)\right\}+O\left(\frac{1}{T}\right) .
$$


Next, using the lower bound in (9), we find that this is

$$
\geq \frac{4}{\pi} \frac{(\sin \delta \theta T / 2)^{2}}{\theta} \frac{\log ^{2} T}{T} \int_{\beta+\eta}^{\beta+2-\eta} F(\alpha, \theta T) d \alpha+o\left(\frac{\log ^{2} T}{T}\right) .
$$

Finally, by (4) we have that

$$
J(\beta+2, T)-J(\beta, T) \geq \frac{4}{\pi} \frac{(\sin \theta / 2)^{2}}{\theta} \frac{\log ^{2} T}{T}\left(2 / 3-c_{1}(\eta)\right)+o\left(\frac{\log ^{2} T}{T}\right) .
$$

The optimal choice of $\theta$ is the unique solution $($ on $(0, \pi))$ of the equation $\tan \theta / 2=\theta$, namely $\theta=2.33112 \ldots$. Using this and taking $\eta$ sufficiently small, we obtain

$$
\begin{aligned}
J(\beta+2, T)-J(\beta, T) & \geq(.307+o(1)) \frac{\log ^{2} T}{T} \\
& \geq .3 \frac{\log ^{2} T}{T},
\end{aligned}
$$

for $\beta>0$ and all $T$ sufficiently large.

To obtain the upper bound we again take $g$ and $\hat{g}$ as above (although $b$ will be different). By the growth condition on $g$ and the estimate $\sum_{u-1<\gamma \leq u} 1 \ll$ $\log (|u|+2)$, we easily obtain the bound

$$
\left|\sum_{\gamma} g\left( \pm(t-\gamma) \frac{\log T}{2 \pi}\right)\right| \ll \log (|t|+2) .
$$

Using this, we find that

$$
\begin{aligned}
\int_{0}^{\infty} & \left(\frac{\sin \frac{\delta}{2} t}{t}\right)^{2}\left|\sum_{\gamma} g\left( \pm(t-\gamma) \frac{\log T}{2 \pi}\right)\right|^{2} d t \\
= & \int_{0}^{T \log ^{3} T}\left(\frac{\sin \frac{\delta}{2} t}{t}\right)^{2}\left|\sum_{\gamma} g\left( \pm(t-\gamma) \frac{\log T}{2 \pi}\right)\right|^{2} d t \\
& +O\left(\int_{T \log ^{3} T}^{\infty} \frac{\log ^{2} t}{t^{2}} d t\right) \\
\leq & \frac{\delta^{2}}{4} \int_{0}^{T}\left|\sum_{\gamma} g\left( \pm(t-\gamma) \frac{\log T}{2 \pi}\right)\right|^{2} d t \\
& +\sum_{k=1}^{[5 \log \log T]} \int_{2^{k-1} T}^{2^{k} T}\left|\sum_{\gamma} g\left( \pm(t-\gamma) \frac{\log T}{2 \pi}\right)\right|^{2} t^{-2} d t+o\left(\frac{1}{T}\right) \\
\leq & \frac{\delta^{2}}{4} H_{ \pm}\left(\frac{\log T}{2 \pi}, T\right)+T^{-2} \sum_{k=1}^{[5 \log \log T]} 2^{2-2 k} H_{ \pm}\left(\frac{\log T}{2 \pi}, 2^{k} T\right) \\
& +o\left(\frac{1}{T}\right) .
\end{aligned}
$$


The bound for $H_{ \pm}$from (9) is applicable in this range of $k$ and leads to

$$
\begin{aligned}
\leq & (1+o(1))\left\{\frac{1}{4 T} \int_{\beta}^{\beta+b} F(\alpha, T) d \alpha+\frac{4}{T} \sum_{k=1}^{[5 \log \log T]} 2^{-k} \int_{\beta}^{\beta+b} F\left(\alpha, 2^{k} T\right) d \alpha\right\} \\
& +o\left(\frac{1}{T}\right) .
\end{aligned}
$$

If we now set $b=2+2 \eta$ and use (5) we find that this is

$$
\begin{aligned}
& \leq(1+o(1))\left\{\frac{4+c_{3}(2 \eta)}{4 T}+\frac{4\left(4+c_{3}(2 \eta)\right)}{T}\right\}+o\left(\frac{1}{T}\right) \\
& \leq \frac{17+5 c_{3}(2 \eta)+o(1)}{T}
\end{aligned}
$$

as $T \rightarrow \infty$. Thus, we have shown that

$$
\begin{aligned}
\int_{0}^{\infty} & \left(\frac{\sin \frac{\delta}{2} t}{t}\right)^{2}\left|\sum_{\gamma} g\left( \pm(t-\gamma) \frac{\log T}{2 \pi}\right)\right|^{2} d t \\
& \leq \frac{17+5 c_{3}(2 \eta)+o(1)}{T}
\end{aligned}
$$

as $T \rightarrow \infty$.

We now combine (8), (10) (with $b=2+2 \eta$ ), and (11) to obtain

$$
\begin{aligned}
J(\beta+ & 2+\eta, T)-J(\beta+\eta, T) \\
\leq & \frac{2}{\pi} \log ^{2} T \int_{0}^{\infty}\left(\frac{\sin \frac{\delta}{2} t}{t}\right)^{2} \\
& \times\left\{\left|\sum_{\gamma} g\left((t-\gamma) \frac{\log T}{2 \pi}\right)\right|^{2}+\left|\sum_{\gamma} g\left((\gamma-t) \frac{\log T}{2 \pi}\right)\right|^{2}\right\} d t \\
& +O\left(\frac{1}{T}\right) \\
\leq & \frac{4}{\pi}\left(17+5 c_{3}(2 \eta)+o(1)\right) \frac{\log ^{2} T}{T} \\
\leq & \left(21.646+7 c_{3}(2 \eta)+o(1)\right) \frac{\log ^{2} T}{T}
\end{aligned}
$$

as $T \rightarrow \infty$. Taking $\eta$ sufficiently small, we see that

$$
J(\beta+2+\eta, T)-J(\beta+\eta, T) \leq 21.65 \frac{\log ^{2} T}{T}
$$

for $\beta>0$ and all sufficiently large $T$. This gives the upper bound and completes the proof of the theorem.

We now prove the corollary. For $\beta>2$ the corollary follows immediately from the theorem. Suppose then that $1 \leq \beta \leq 2$. By (2) and the fact that $J(\beta, T)$ is an increasing function of $\beta$ we have

$$
\frac{1}{2} \frac{\log ^{2} T}{T} \sim J(1, T) \leq J(\beta, T) \leq J(3, T) .
$$


Thus,

and, by the theorem,

$$
J(\beta, T) \gg \frac{\log ^{2} T}{T} \gg \beta \frac{\log ^{2} T}{T}
$$

$$
\begin{gathered}
J(\beta, T) \leq(J(3, T)-J(1, T))+J(1, T) \\
\ll \frac{\log ^{2} T}{T} \ll \beta \frac{\log ^{2} T}{T} . \\
\text { REFERENCES }
\end{gathered}
$$

\section{REFERENCES}

1. P. X. Gallagher and J. Mueller, Primes and zeros in short intervals, J. Reine Angew. Math. 303/304 (1978), 205-220.

2. D. A. Goldston, On the function $S(T)$ in the theory of the Riemann zeta-function, J. Number Theory 27 (1987), 149-177.

3. $\ldots$, On the pair correlation conjecture for zeros of the Riemann zeta-function, J. Reine Angew. Math. 385 (1988), 24-40.

4. D. A. Goldston and S. M. Gonek, Mean values of $\zeta^{\prime} / \zeta(s)$ and primes in short intervals, (in preparation).

5. D. A. Goldston and H. L. Montgomery, Pair correlation of zeros and primes in short intervals, in Analytic Number Theory and Diophantine Problems, Proc. of a conference at Oklahoma State University, 1984, Birkhäuser, Boston-Basel-Stuttgart 1987, 183-203.

6. H. L. Montgomery, The pair correlation of zeros of the zeta function, Proc. Symp. Pure Math. 24 (1973), 181-193.

7. A. Selberg, On the normal density of primes in small intervals, and the difference between consecutive primes, Arch. Math. Naturvid. 47 (1943), 87-105.

Department of Mathematics and Computer Science, San Jose State University, San Jose, California 95192

Department of Mathematics, University of Rochester, Rochester, New York 14627 